\title{
Time-Reversed OFDM Communication in Underwater Channels
}

\author{
João Gomes ${ }^{1} \quad$ Victor Barroso \\ Instituto Superior Técnico - Instituto de Sistemas e Robótica \\ Av. Rovisco Pais, 1049-001 Lisboa, Portugal \\ $\{$ jpg,vab\}@isr.ist.utl.pt
}

\begin{abstract}
Time reversal is a feedback wave focusing technique that can be used to transparently compensate for multipath distortion in digital communications over several types of physical propagation media, such as radio or acoustic channels. While much of the work on this topic has focused on coherent communication through single-carrier modulation, time reversal can in fact be applied to any signaling scheme. This paper discusses issues related to protocol design and data modulation/demodulation when multicarrier signals are used, with an emphasis on underwater communication. It is shown that focusing information can be derived at the transmitter array by prefiltering a single observed broadband channel probe, thus streamlining the design of communication protocols. Conventional prefix-based demodulation can be achieved for sufficiently well focused OFDM signals, but this may be somewhat demanding in terms of transmitter hardware. A receiver architecture based on multipleinput/multiple-output decision-feedback equalization is proposed when few transmit elements are used, creating significant residual intersymbol interference.
\end{abstract}

\section{INTRODUCTION}

Time reversal, or broadband phase conjugation, is a feedback technique that takes advantage of the physics of wave propagation to focus signals at desired points in a waveguide with high energy and low distortion [1]. Its potential in underwater communications aroused much interest $[2,3]$ after the general concept was successfully demonstrated in the ocean [1]. This approach is currently being considered in the context of radio communications as well.

Time-reversed communication uses an array of transducers, known as a time-reversal mirror to perform transmitter-side broadband beamforming. The spatial information needed at the mirror is acquired during the forward phase of communication, when the intended receiver transmits a channel probe whose distorted replicas encode the multipath structure of the channel. By reversing those signals in time, modulating them with an information waveform, and transmitting them during the reciprocal phase, the mirror generates an acoustic field that retraces the original one, such that all the replicas traveling in the water simultaneously converge on the receiver. This generates a strong signal with low intersymbol interference (ISI) that substantially eases the equalization and tracking effort needed by a coherent receiver to operate reliably at high data rates [4]. In line with recent trends in wireless communications [5],

\footnotetext{
${ }^{1}$ This work was partially supported by the FCT Programa Operacional Sociedade da Informação (POSI) in the frame of QCA III, under contract POSI/CPS/33205/2000.
}

multipath is regarded here as beneficial, providing multiple interfering beams at the focus that increase the SNR and help to stabilize the acoustic field in the presence of fluctuations in the environment.

Incoherent communication based on M-FSK modulation has been widely used in medium-speed underwater data links due to its resilience to multipath [4]. Coherent multicarrier modulation, on the other hand, has received scarce attention $[6,7]$, which contrasts sharply with the large body of work on this subject in wireline and wireless radio communications, where orthogonal frequency-division multiplexing (OFDM) is one of the preferred ways of approaching capacity in frequency-selective channels. This discrepancy may be due to the perception that most off-vertical underwater channels are too complex and dynamic for the subtle orthogonality of carriers to be ensured reliably. In the context of time-reversed focusing, however, the situation may be somewhat different due to the consistency and low delay distortion of the acoustic field near the focal spot. Based on that premise, the goal of the present work is to investigate, through simulation, modulation and demodulation strategies for time-reversed OFDM communication under static conditions.

Regarding modulation, a protocol is proposed that allows the mirror to acquire a signal basis needed for regenerating arbitrary OFDM waveforms at the focus. A straightforward extension of the single-carrier case [2] would require sequentially transmitting the various subcarrier signaling pulses as channel probes during the forward phase, a somewhat cumbersome procedure. Here, a faster alternative is developed, which consists in sending a single broadband pulse, or a known data packet, that covers the whole available bandwidth. From the received waveform, a signal basis can be derived by bandpass filtering at the mirror. This type of transmit prefiltering is very simple, and can be shown to possess interesting optimality properties [2].

OFDM demodulation based on the insertion of cyclic prefixes, Fourier synthesis/analysis and per-tone frequency equalization is a very appealing strategy, but cannot realistically be applied in arbitrary underwater channels with extremely long impulse responses. By contrast, it seems like a feasible approach to be used at the focus, given the time compression attained during the reciprocal phase. The performance of this type of receiver is compared to an alternative one based on multipleinput/single-output decision-feedback equalization.

Notation: Throughout the paper, vectors and matrices are represented by lowercase and uppercase boldface letters, respectively. The superscript $(\cdot)^{T}$ denotes transpose, and $(\cdot)^{*}$ stands for conjugate transpose (hermitian) of matrices, vectors or scalars. Convolution is denoted by $*$.

\section{Pulse Reshaping and Time Reversal}

When a communications waveform $x(t)$ is transmitted $^{2}$ from

\footnotetext{
${ }^{2}$ Most of the signals in this paper are bandpass, and will be represented by their lowpass complex envelopes.
} 
the focus, the received signal at the $m$-th mirror transducer in the absence of noise is given by the convolution

$$
y_{m}(t)=x(t) * g_{m}(t)
$$

where $g_{m}(t)$ is the medium impulse response between the focal point and the transducer. If subsequently the time-reversed signals $y_{m}^{*}(-t)=x^{*}(-t) * g_{m}^{*}(-t)$ are simultaneously transmitted, then by reciprocity the observed signal at the focus satisfies

$$
z(t)=\sum_{m} y_{m}^{*}(-t) * g_{m}(t)=x^{*}(-t) * \sum_{m} g_{m}^{*}(-t) * g_{m}(t),
$$

or, in the frequency domain,

$$
Z(\omega)=X^{*}(\omega) \sum_{m}\left|G_{m}(\omega)\right|^{2}
$$

If the mirror is sufficiently long and dense to capture a significant fraction of the acoustic energy transmitted by the source, then wave refocusing ensures that $z(t) \approx x^{*}(-t)$. In turn, this implies that $\sum_{m}\left|G_{m}(\omega)\right|^{2}$ is approximately constant in the signal band.

To effectively convey digital messages, a time-reversal mirror must first acquire a signal basis, i.e., a set of waveforms that can be combined to regenerate an arbitrary information-bearing digital signal with low distortion at the focus. The communication protocol proposed in [2] for PAM modulation accomplishes this simply by transmitting a single signaling pulse to the mirror. Due to the linearity of this type of modulation, the mirror can focus any desired PAM waveform if it responds with a train of time-reversed and delayed replicas of the received distorted pulses, weighted by the symbols of a signal constellation.

In [2] a strategy for reducing the bandwidth (and data rate) of PAM signals during the reciprocal phase, while preserving the original focusing information, was proposed. It simply involves inserting at each mirror transducer a pulse shaping filter that would convert the forward, broader bandwidth, pulse to the desired one in the absence of channel distortion. As detailed next, the same principle can be applied under more general conditions.

Suppose that a pulse $f(t)$, occupying a frequency band $W_{f}$, is transmitted and its distorted replicas $h_{m}(t)=f(t) * g_{m}(t)$ are received and recorded at the mirror. If subsequently one seeks to focus a signal $x(t)$ such that $(i)$ its spectral support $W_{x}$ is contained in $W_{f}$, and $(i i) F(\omega)$ is flat over $W_{x}$, then it is possible to reuse the implicit environmental information gained from $f(t)$ by transmitting $y_{m}(t)=x(t) * h_{m}^{*}(-t)$. Similarly to $(2)$, the refocused signal is given by

$$
\begin{aligned}
z(t) & =\sum_{m} y_{m}(t) * g_{m}(t) \\
& =x(t) * f^{*}(-t) * \sum_{m} g_{m}^{*}(-t) * g_{m}(t), \\
Z(\omega) & =X(\omega) F^{*}(\omega) \sum_{m}\left|G_{m}(\omega)\right|^{2} .
\end{aligned}
$$

Once again, $\sum_{m}\left|G_{m}(\omega)\right|^{2}$ is approximately constant in $W_{x} \subset$ $W_{f}$, and by design $X(\omega) F^{*}(\omega) \propto X(\omega)$. Even if the latter condition is not met, the frequency distortion introduced by $F(\omega)$ can be compensated at the receiver by postfiltering since $W_{x}$ and $F(\omega)$ are known, as long as $F(\omega) \neq 0, \forall \omega \in W_{x}$.

\section{OFDM MODULATION}

The approach described in Section II allows a signal basis to be extracted from a broadband pulse for any type of bandlimited modulation. This work is particularly concerned with OFDM modulated signals, which can be viewed as a superposition of PAM waveforms

$$
x(t)=\sum_{i=1}^{M} \sum_{k} a_{i}(k) f_{i}\left(t-k T_{b}\right),
$$

where $a_{i}(k)$ denotes a symbol from the $i$-th subcarrier complex constellation, $f_{i}(t)$ is the corresponding pulse shape, and $T_{b}$ is the symbol interval. The pulses $f_{i}(t)$ are commonly generated by exponential modulation from a single rectangular prototype with duration $T_{b}, \Pi\left(t / T_{b}\right)$, as

$$
f_{i}(t)=e^{j \frac{2 \pi i}{M} \cdot \frac{t}{T_{b}}} \Pi\left(\frac{t}{T_{b}}\right) .
$$

This type of modulation has aroused much interest in wireless radio communications due to the simplicity of the demodulator [5]. If $x(t)$, defined by (6)-(7), is transmitted through a SISO time-invariant channel whose impulse response $g(t)$ has length $L_{g}$, then it is possible to recover the $k$-th block of $M$ symbols, $\left\{a_{i}(k)\right\}_{i=1}^{M}$, by Fourier analysis if

1. A cyclic prefix with duration $T_{c} \geq L_{g}$ is added to each symbol, i.e., (7) is augmented before $t=0$ with a copy of $f_{i}(t), t \in\left[T_{b}-T_{c}, T_{b}\right)$, and in (6) $T_{b}$ is then adjusted to $T_{c}+T_{b}$. This prefix allows each subcarrier signal (a pure tone) to attain steady state before Fourier analysis at the receiver.

2. The channel transfer function $G(\omega)$ does not have zeros at the subcarrier frequencies.

Due to the long impulse responses that are typically observed in underwater channels, cyclic prefix insertion is unfeasible in practice because it would drastically reduce the data rate. That, however, may not be the case when time reversal is used, as the effective length of the overall impulse response experienced at the focal spot may be much shorter than those measured at the mirror sensors during the forward phase. Still, one must keep in mind that residual ISI components due to imperfect focusing may span intervals longer than one OFDM symbol.

Following the approach of Section II, the OFDM signal $x(t)$ in (6) could be generated with ideal pulse shapes, and then filtered by the distorted probes $h_{m}^{*}(-t)$. Equivalently, distorted subcarrier pulses could be obtained by reshaping for each mirror transducer, $f_{i m}^{\prime}(t)=f_{i}(t) * h_{m}^{*}(-t)$, and then modulated by the data to directly generate the $m$-th transmitted signal $y_{m}(t)$.

The $h_{m}(t)$ could be observed directly at the mirror from a single broadband transmitted pulse, but for noise robustness (and least-squares optimality [2]) a better option is to estimate them from a longer data packet, originating at the focus, that acts as a channel probe. Note that this packet could be modulated not only in OFDM, but in any other format, as long as its spectral content is sufficiently rich. The issue of probe design for underwater channel estimation and OFDM symbol synchronization is considered in [6], where a pseudo-random Gaussian noise (PRGN) sequence is proposed as an optimal OFDM sounding waveform with nearly constant spectral envelope across the desired bandwidth and extremely low sidelobe levels.

\section{Data Model for Equalization}

This work adopts the discrete-time redundant filterbank approach of [8] that includes OFDM transmission as a special case. 
It provides an elegant framework for analyzing the impact of the channel and subcarrier pulse shapes on symbol estimates.

Based on a vector of $M$ input symbols at time $n$,

$$
\overline{\mathbf{a}}(n)=\left[a_{1}(n) \ldots a_{M}(n)\right]^{T},
$$

the transmitter generates a vector (or block) $\overline{\mathbf{x}}(n)$ of $P \geq M$ samples by convolution with a linear precoder

$$
\overline{\mathbf{x}}(n)=\mathbf{F} * \overline{\mathbf{a}}(n),
$$

where $\mathbf{F}$ denotes a sequence of $P \times M$ matrices $\mathbf{F}_{n}$. This vector is transmitted through a channel whose impulse response is represented by the (FIR) sequence of $P \times P$ matrices $\mathbf{G}_{n}$, generating

$$
\overline{\mathbf{y}}(n)=\mathbf{G} * \overline{\mathbf{x}}(n)+\overline{\mathbf{v}}(n),
$$

where $\overline{\mathbf{v}}(n)$ denotes an additive noise vector. The transmission channel underlying $\mathbf{G}$ is assumed to be MIMO with $n_{i}$ inputs and $n_{o}$ outputs. The coded vector $\overline{\mathbf{x}}$ therefore comprises $n_{i}$ streams, each containing $P^{\prime}=P / n_{i}$ samples to be sequentially fed to one of the channel inputs after parallel to serial conversion. Each $\mathbf{G}_{n}$ in (10) interleaves $n_{i} n_{o}$ convolution matrices that model the distortion introduced by the channel in a block of $P^{\prime}$ samples between every pair of input-output terminals. The specific layout of samples inside $\overline{\mathbf{x}}$ and $\overline{\mathbf{y}}$ is of little relevance and will not be detailed here. Note that the MIMO framework can model the existence of multiple physical transmit and receive elements, as well as virtual (output) channels such as the polyphase components of fractionally-sampled cyclostationary communications waveforms. It can be used to characterize the demodulation of data during both the forward and reciprocal phases of time-reversed communication.

When the channel impulse response duration does not exceed one OFDM symbol interval, only two of the $\mathbf{G}_{n}$ in (10) are nonzero. Adding a cyclic prefix or trailing zeros as part of the (memoryless) precoding process exploits the structure of these matrices to reduce the demodulation problem to an equivalent one where interblock interference is absent [8]. As discussed in Section III, that assumption may be violated even when time reversal is used, and this work therefore investigates generic MIMO equalization as a means of demodulating data blocks.

At the receiver, $N \triangleq N_{2}-N_{1}+1$ channel outputs are stacked ${ }^{3}$ to form a larger equalizer input vector

$$
\mathbf{y}(n)=\left[\overline{\mathbf{y}}^{T}\left(n-N_{1}\right) \ldots \overline{\mathbf{y}}^{T}\left(n-N_{2}\right)\right]^{T},
$$

This vector may be related to the transmitted data blocks by first defining the combined channel-precoder response

$$
\mathbf{H}_{n}=\sum_{i} \mathbf{G}_{i} \mathbf{F}_{n-i}
$$

which is assumed to be an FIR sequence of $P \times M$ matrices for $\mu \leq n \leq \nu$. Then, (11) is written as

$$
\mathbf{y}(n)=\mathbf{H a}(n)+\mathbf{v}(n),
$$

where $\mathbf{H}$ is the block Toeplitz MIMO channel convolution matrix

$$
\mathbf{H}=\left[\begin{array}{ccccccc}
\mathbf{H}_{\mu} & \mathbf{H}_{\mu+1} & \ldots & \mathbf{H}_{\nu} & \mathbf{0} & \ldots & \mathbf{0} \\
\mathbf{0} & \mathbf{H}_{\mu} & \mathbf{H}_{\mu+1} & \ldots & \mathbf{H}_{\nu} & \mathbf{0} & \ldots \\
\vdots & & \ddots & & & \ddots & \vdots \\
\mathbf{0} & \ldots & \mathbf{0} & \mathbf{H}_{\mu} & \mathbf{H}_{\mu+1} & \ldots & \mathbf{H}_{\nu}
\end{array}\right]
$$

\footnotetext{
${ }^{3}$ Normally, one would like $\mathbf{y}(n)$ to have both causal and anticausal components, hence $N_{1} \leq 0, N_{2} \geq 0$.
}

$$
\begin{aligned}
\mathbf{a}(n) & =\left[\begin{array}{lll}
\overline{\mathbf{a}}^{T}\left(n-N_{1}-\mu\right) & \ldots \overline{\mathbf{a}}^{T}\left(n-N_{2}-\nu\right)
\end{array}\right]^{T} \\
\mathbf{v}(n) & =\left[\begin{array}{lll}
\overline{\mathbf{v}}^{T}\left(n-N_{1}\right) \ldots \overline{\mathbf{v}}^{T}\left(n-N_{2}\right)
\end{array}\right]^{T} .
\end{aligned}
$$

As noted in [8], the introduction of redundancy through linear precoding causes matrix $\mathbf{H}$ to become tall if a sufficient number of channel observations are stacked in $\mathbf{y}$. By properly choosing the precoder, $\mathbf{H}$ may become full column rank as well, and hence left invertible. Related results are presented in [9], where the redundancy gained by suppressing some of the OFDM tones at the transmitter allows the receiver to perfectly recover $\overline{\mathbf{a}}$ (zeroforcing equalization) even without the introduction of a cyclic prefix.

Channel invertibility issues are certainly relevant, but will not be addressed in this work. Here, the precoder $\mathbf{F}$ is used mainly as a tool to format the subcarrier signaling pulses $f_{i}$ so that their spectra can tightly fit the scarce available bandwidth (unlike the sinc spectra of rectangular pulses), and to reduce their spectral overlap [10]. The latter aspect should play an important role in minimizing intercarrier interference (ICI) caused by channel variations.

Given the observation (13), a MIMO MMSE decisionfeedback equalizer (DFE) based on channel estimation will be used to recover $\overline{\mathbf{a}}(n)$ as follows [11]. Partition the channel matrix as $\mathbf{H}=\left[\begin{array}{l}\mathbf{H}_{f} \\ \mathbf{H}_{b}\end{array}\right]$, such that the lower right block in $\mathbf{H}_{f}$ is $\mathbf{H}_{-N_{2}}$. Accordingly, partition the data vector as $\mathbf{a}^{T}(n)=\left[\mathbf{a}_{f}^{T}(n) \mathbf{a}_{b}^{T}(n)\right]^{T}$, where $\mathbf{a}_{b}(n)$ contains the past data blocks $\overline{\mathbf{a}}(n-1), \ldots, \overline{\mathbf{a}}\left(n-N_{2}-\nu\right)$ that contribute to $\mathbf{y}(n)$ and which are fully known at time $n$, assuming that perfect decisions are made. From $\mathbf{y}(n), \mathbf{a}_{b}(n)$, and an estimate of the causal channel components which make up $\mathbf{H}_{b}$, generate the modified equalizer input vector

$$
\mathbf{y}_{f}(n)=\mathbf{y}(n)-\mathbf{H}_{b} \mathbf{a}_{b}(n),
$$

from which all known postcursor ISI has been removed. The estimated data block is given by

$$
\hat{\overline{\mathbf{a}}}(n)=\mathbf{W}_{f}^{*} \mathbf{y}_{f}(n)-\left(\mathbf{B}_{0}-\mathbf{I}_{M}\right)^{*} \overline{\mathbf{a}}(n),
$$

where $\mathbf{I}_{M}$ stands for the $M \times M$ identity matrix and $\mathbf{W}_{f}, \mathbf{B}_{0}$ are the feedforward and feedback coefficient matrices. Denoting the covariances of $\mathbf{a}_{f}(n)$ and $\mathbf{v}(n)$ by $\mathbf{R}_{a_{f} a_{f}}$ and $\mathbf{R}_{v v}$, the feedback matrix is obtained from the Cholesky decomposition of the auxiliary matrix

$$
\mathbf{R} \triangleq \mathbf{R}_{a_{f} a_{f}}^{-1}+\mathbf{H}_{f}^{*} \mathbf{R}_{v v}^{-1} \mathbf{H}_{f}, \quad \mathbf{R}=\mathbf{L D L}^{*},
$$

where $\mathbf{L}$ is a lower triangular Cholesky factor with unit diagonal, and $\mathbf{D}$ is diagonal. Then, $\mathbf{B}_{0}$ equals the lower right $M \times M$ block of $\mathbf{L}$, and its lower triangular structure ensures that (18) is computable if the elements of $\overline{\mathbf{a}}(n)$ are sequentially decoded from $a_{M}(n)$ down to $a_{1}(n)$. Finally,

$$
\mathbf{W}_{f}=\mathbf{R}_{v v}^{-1} \mathbf{H}_{f} \mathbf{R}^{-1} \mathbf{B},
$$

where $\mathbf{B}$ is formed from the rightmost $M$ columns of $\mathbf{L}$.

The adaptive implementation of this type of equalizer is discussed in [11]. Related results on adaptive multiuser decisionfeedback equalization under severe ISI can be found in [12], although the emphasis of that work on user discrimination by spatial processing at the receiver is unsuited for (MISO) timereversed focusing. 


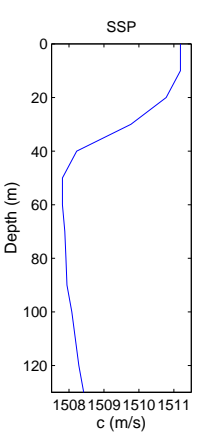

(a)

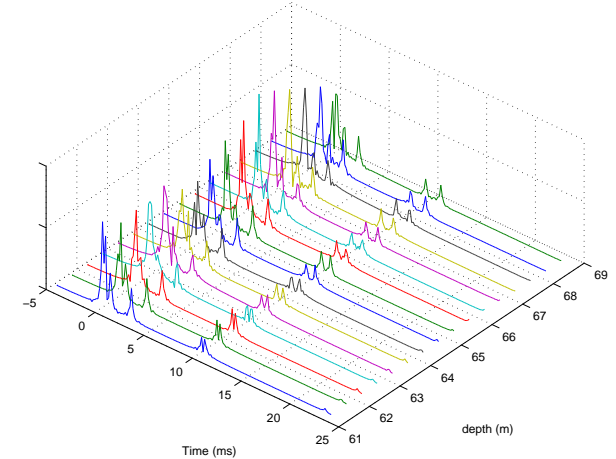

(b)
Figure 1: Simulated environment (a) Sound-speed profile (b) Received probe magnitudes
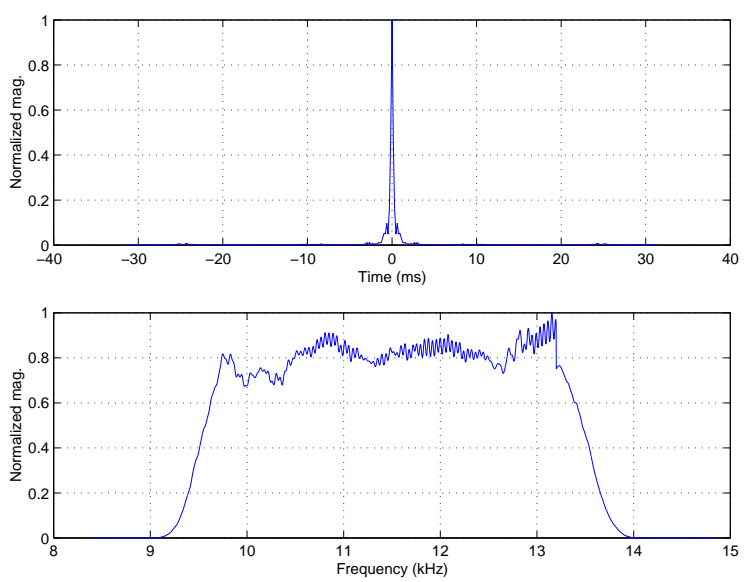

Figure 2: Equivalent probe shapes at the focus (a) Time domain (b) Frequency domain

\section{Simulation Results}

The simulated environment is a range-independent ocean cross-section with $130 \mathrm{~m}$ depth, $60 \mathrm{~m}$ source depth and $2 \mathrm{Km}$ source-array range. Acoustic propagation was simulated using a Gaussian beam ray tracer with sound-speed profile shown in Figure 1a, constant bottom reflection coefficient $\alpha_{B}=0.6$, and surface reflection modeled as a deterministic angle-dependent coefficient equal to the average specular component for a surface RMS roughness of $0.4 \mathrm{~m}$ [13]. The mirror is a vertical array with 16 transducers spaced $0.25 \mathrm{~m}$ apart from depth $61 \mathrm{~m}$ to $68.5 \mathrm{~m}$.

The channel probe is a single raised-cosine pulse with $20 \%$ rolloff and flat spectrum between $9.8 \mathrm{kHz}$ and $13.3 \mathrm{kHz}$. Figure $1 \mathrm{~b}$ shows the magnitudes of the distorted pulses received at the array, with delay spreads in excess of $10 \mathrm{~ms}$. The path delays have been normalized so that the earliest replica arrives at time zero. Figure 2 shows the equivalent regenerated pulse at the focal spot computed from (3). Some minor multipath components persist at delays $\pm 10 \mathrm{~ms}$ and $\pm 25 \mathrm{~ms}$, but the original pulse has essentially been compressed back to a main lobe width of about $0.5 \mathrm{~ms}$.

Prefix-based demodulation Under the favorable conditions of Figure 2, prefix-based OFDM demodulation seems feasible. Table 1 lists the parameters of the simulated OFDM

\begin{tabular}{||l|l||l|l||}
\hline \hline Subcarriers & 28 & Time redundancy & $32 / 28$ \\
Lower frequency & $10 \mathrm{kHz}$ & Carrier spacing & $718 \mathrm{~Hz}$ \\
Constellations & $\{-1,+1\}$ & Symbol duration & $10 \mathrm{~ms}$ \\
Oversampling & 2 & & \\
\hline \hline
\end{tabular}

Table 1: OFDM parameters

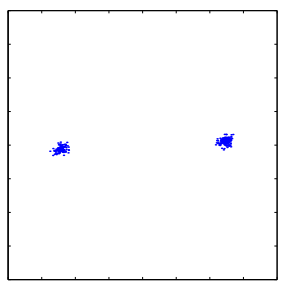

(a)

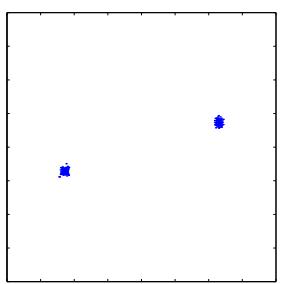

(b)

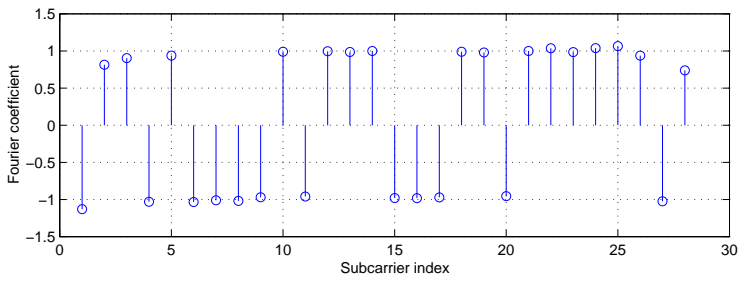

Figure 3: Estimated subcarrier symbols for prefix-based demodulation with (16-element mirror) (a) Subcarrier \#3 (b) Subcarrier \#19 (c) Output snapshot

waveform using a rectangular prototype. In this setup, the time redundancy parameter is the ratio between the duration of an OFDM symbol with and without inclusion of a cyclic prefix. Perfect Fourier-based demodulation should therefore be accomplished if the overall refocused impulse response duration does not exceed about $1.5 \mathrm{~ms}$. Figure 3 depicts two of the raw subcarrier constellations, and one snaphot of the estimated data block $\hat{\mathbf{a}}(n)$ after magnitude compensation ${ }^{4}$.

To test this approach under more demanding conditions, the number of mirror transducers was decimated by a factor of 4 , which further limits its focusing power. Figure 4 shows a clear degradation in demodulation performance, although the error probability may still be deemed acceptable.

MIMO Decision-Feedback Equalization: A MIMO DFE was used to reduce the residual symbol estimation errors under sparse focusing with a 4-element mirror. In this framework, there is no obvious advantage in retaining rectangular subcarrier pulse shapes, so the precoder $\mathbf{F}_{n}$ in (9) was instead based on a raised-cosine prototype that provides more effective spectral containment. Its zeros are spaced at the OFDM symbol rate, and the rolloff factor is $50 \%$. To preserve the level of time redundancy given in Table 1 the precoding matrices are $32 \times 28$.

At the receiver, the OFDM waveform was oversampled by a factor of 2 . This is best modeled by splitting the scalar discretetime signal into 2 polyphase components, so that 64 samples are collected per symbol interval and the equivalent MIMO system is $64 \times 28$. Most of the energy in the overall impulse response $\mathbf{H}_{n}$ is concentrated between block indices $\mu=-2$ and $\nu=2$.

\footnotetext{
${ }^{4}$ In this setup, simple division by the channel gains at the subcarrier frequencies leaves residual phase and magnitude offsets in several subconstellations. Therefore, the $a_{i}(n)$ were postprocessed to ensure that $\left|\hat{a}_{i}(n)\right|=1$ on average.
} 


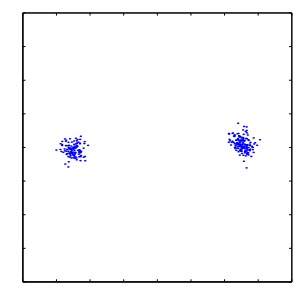

(a)

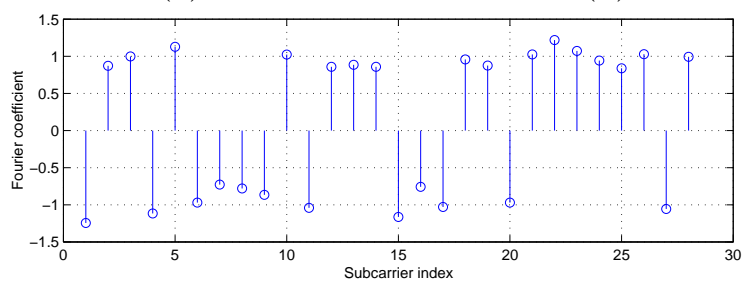

Figure 4: Estimated subcarrier symbols for prefix-based demodulation (4-element mirror) (a) Subcarrier \#3 (b) Subcarrier \#19 (c) Output snapshot

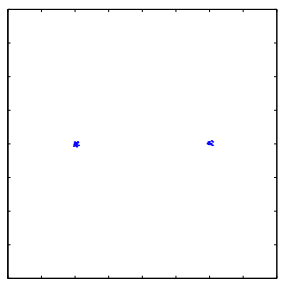

(a)

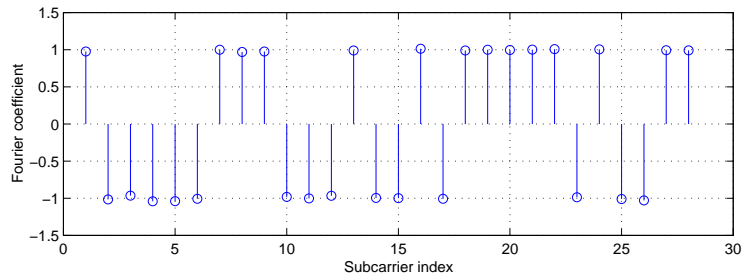

Figure 5: Estimated subcarrier symbols for MIMO DFEbased demodulation (4-element mirror) (a) Subcarrier \#3 (b) Subcarrier \#19 (c) Output snapshot

Finally, $N_{1}=-2, N_{2}=1$ were used to build the $256 \times 1$ equalizer input vector (11). This choice of $N_{1}$ captures in $\mathbf{y}(n)$ most of the precursor energy from $\overline{\mathbf{a}}(n)$.

Figure 5 shows some of the equalizer output values under noiseless conditions and assuming perfect channel knowledge. The mean subcarrier MSE is $-30 \mathrm{~dB}$, an improvement of approximately $15 \mathrm{~dB}$ relative to the results of Figure 4 .

\section{Conclusion}

This work analyzed a simple but general method for deriving focusing information from a broadband channel probe in time-reversed communications. The overhead imposed on communication protocols to convey a signal basis during the forward phase is thereby minimized, as all waveforms can be extracted from a single suitably designed probe, regardless of the complexity of the signal space used during the reciprocal phase. The technique was applied to time-reversed OFDM transmission, and simulation results suggest that residual intersymbol interference can be reduced to a point were conventional demod- ulation based on cyclic prefixes and Fourier analysis becomes feasible with moderate hardware complexity at the transmitter.

OFDM decoding using a MIMO DFE was proposed as an alternative to prefix-based demodulation, which experiences significant degradation when the mirror is too sparse. Even with imperfect focusing, time-reversed communication makes sense because the equalizer may be far less complex than in arbitrary ocean channels. The MIMO DFE proved to be effective, drastically reducing the residual mean-square error with limited complexity. In fact, the computational cost per subcarrier symbol is comparable to that of single-carrier equalizers.

\section{REFERENCES}

[1] W. Kuperman et al., "Phase conjugation in the ocean: Experimental demonstration of an acoustic time-reversal mirror," $J$. Acoust. Soc. Am., vol. 103, pp. 25-40, Jan. 1998.

[2] J. Gomes and V. Barroso, "Asymmetric underwater acoustic communication using a time-reversal mirror," in Proc. of OCEANS'00, Providence, USA, Sept. 2000.

[3] G. Edelmann et al., "Underwater acoustic communication using time reversal," in Proc. of OCEANS'01, Honolulu, USA, Nov. 2001.

[4] D. Kilfoyle and A. Baggeroer, "The state of the art in underwater acoustic telemetry," IEEE J. of Oceanic Eng., vol. 25, pp. 4-27, Jan. 2000

[5] A. Paulraj, R. Nabar, and D. Gore, Introduction to Space-Time Wireless Communications. Cambridge University Press, 2003.

[6] W. Lam and R. Ormondroyd, "A broadband UWA communications system based on COFDM modulation," in Proc. of OCEANS'97, Halifax, Canada, vol. 2, , pp. 862-869, Oct. 1997.

[7] W. Lam, R. Ormondroyd, and J. Davies, "A frequency domain adaptive coded decision-feedback equalizer for a broadband UWA COFDM system," in Proc. of OCEANS'98, Nice, France, Sept. 1998.

[8] A. Scaglione et al., "Linear precoding for estimation and equalization of frequency-selective channels," in Sig. Proc. Advances in Wireless \& Mobile Comm., vol. 1, pp. 291-337, PTR PrenticeHall, 2001.

[9] S. Trautmann and N. Fliege, "Perfect equalization for DMT systems without guard interval," IEEE J. Sel. Areas in Comm., vol. 20, pp. 987-996, June 2002.

[10] G. Cherubini et al., "Filter bank modulation techniques for very high-speed digital subscriber lines," IEEE Comm. Mag., vol. 38, pp. 98-104, May 2000.

[11] J. Gomes and V. Barroso, "MIMO decision-feedback equalization with direct channel estimation," Submitted to SPAWC'2004.

[12] M. Stojanovic and Z. Zvonar, "Multichannel processing of broadband multiuser communication signals in shallow water acoustic channels," IEEE J. of Oceanic Eng., vol. 21, pp. 156-166, Apr. 1996.

[13] L. Brekhovskikh and Y. Lysanov, Fundamentals of Ocean Acoustics. Berlin: Springer-Verlag, second ed., 1991. 